\title{
Beyond the eikonal model for few-body systems
}

\author{
J. S. Al-Khalili, J. A. Tostevin, and J. M. Brooke \\ Department of Physics, University of Surrey, Guildford, Surrey, GU2 5XH, United Kingdom
}

(Received 10 December 1996)

\begin{abstract}
A physical prescription to improve the accuracy of few-body Glauber model calculations of reactions involving loosely bound projectiles is presented in which the eikonal phase shift function of each projectile constituent is modified to account for curvature of its trajectory. Noneikonal effects due to both nuclear and Coulomb interactions are treated on an equal footing. The proposed method is assessed quantitatively by comparison with full quantum mechanical calculations in the case of ${ }^{11} \mathrm{Be}+{ }^{12} \mathrm{C}$ elastic scattering, treated as a three-body ${ }^{10} \mathrm{Be}+n+$ target problem, at energies of 25 and $49.3 \mathrm{MeV} /$ nucleon. Calculated cross-section angular distributions which include the noneikonal modifications are shown to be accurate to larger scattering angles, and for lower incident projectile energies. [S0556-2813(97)50603-0]
\end{abstract}

PACS number(s): 21.45.+v, 24.50.+g, 25.60.-t, 25.70.Bc

The semiclassical eikonal approximation to high-energy projectile scattering has been applied extensively in nuclear physics. Most recently, methods based on the eikonal approximation have formed the basis of few-body calculations of reactions involving the elastic scattering [1,2] and breakup [3] of loosely bound exotic nuclei. While essentially exact calculational schemes have been developed for treating effective three-body systems, e.g. [4], the eikonal models currently provide the only practical methods for quantitative investigations of effective four or more body systems, such as are required to model ${ }^{11} \mathrm{Li}$ or ${ }^{8} \mathrm{He}$ induced reactions.

The resulting simplifications to the quantum few-body problem stem from two sources. The first is the eikonal approximation, in which the incident particles are assumed to follow straight line paths through the interaction field of the target. The second is an adiabatic treatment of the internal degrees of freedom of the composite. We discuss, and make use of, the adiabatic treatment in the following. The present work, however, deals only with corrections to the former, eikonal, approximation. Eikonal models have many variants, the most successful having been formulated by Glauber [5].

Given the economy of the eikonal calculational schemes, many attempts have been made to extend their range of validity by including correction terms. These account for the bending of the path of the particle during the interaction. Saxon and Schiff [6] replaced the eikonal phase by the Wentzel-Kramers-Brillouin (WKB) phase, on the grounds that the latter included the eikonal phase, plus higher-order terms, when expanded in powers of the interaction [7]. Other approaches were reported $[8,9]$ culminating in the work of Wallace [10]. The resulting corrections were expressed as an expansion with the eikonal phase as the leading term.

A quite different prescription, applicable in heavy-ion scattering, where the Coulomb interaction plays a very significant role, as would be indicated by a Sommerfeld parameter $\eta \gg 1$, was proposed by Vitturi and Zardi [11].

All previous noneikonal discussions have been confined to a consideration of structureless point particle scattering from a target potential. In this Rapid Communication our aim is to present and assess a prescription to include the most important noneikonal corrections for a composite few-body projectile, and so to improve the accuracy of reaction observables calculated using few-body Glauber (FBG) models.
Specifically, we are interested in applications to reactions of light composite projectiles such as ${ }^{6} \mathrm{He},{ }^{8} \mathrm{~B},{ }^{11} \mathrm{Be},{ }^{11} \mathrm{Li}$, etc., comprising a core nucleus and one or more loosely bound valence nucleons, at incident energies of a few 10's of $\mathrm{MeV}$ per nucleon. Data for such systems are now being accumulated with ever increasing accuracy at several facilities worldwide. Such projectiles, some with neutron-target subsystems, and all with core-target Sommerfeld parameters $\eta_{c}<1$, are not amenable to the simple Vitturi-Zardi prescription and require a consideration of corrections to the eikonal phase for each projectile constituent-target two-body subsystem which enters the few-body reaction amplitude.

For composite $n$-body nuclei (where the $n$ bodies are core clusters or individual nucleons) the FBG model makes the adiabatic approximation [12], freezing the internal coordinates of the projectile constituents during their passage by the target. Thus each constituent is assumed to interact independently with the target via a two-body interaction or optical potential, and to follow its own straight line path through the interaction region; the eikonal aspect. We consider noneikonal corrections, the bending of these individual trajectories, due to both the nuclear and Coulomb interactions. We will present calculated cross-section angular distributions for ${ }^{11} \mathrm{Be}+{ }^{12} \mathrm{C}$ elastic scattering at 25 and $49.3 \mathrm{MeV} /$ nucleon, an effective three-body ${ }^{10} \mathrm{Be}+n+{ }^{12} \mathrm{C}$ system. In such threebody cases full quantum mechanical calculations, which make the adiabatic approximation but not the additional eikonal assumption, can be performed and so can be used to assess the importance and validity of the noneikonal modifications made to the FBG model.

The adiabatic approximation to the Schrödinger equation for the scattering from a structureless target of a $n$-body projectile, with internal Hamiltonian $H_{0}$, ground state wave function $\Phi_{0}\left(\vec{r}_{1}, \ldots, \vec{r}_{n}\right)$ and energy $\varepsilon_{0}$, is

$$
\begin{aligned}
& {\left[T_{R}+\varepsilon_{0}+U\left(\vec{R}, \vec{r}_{1}, \ldots, \vec{r}_{n}\right)\right] \Psi_{\vec{K}}\left(\vec{R}, \vec{r}_{1}, \ldots, \vec{r}_{n}\right)} \\
& \quad=E \Psi_{\vec{K}}\left(\vec{R}, \vec{r}_{1}, \ldots, \vec{r}_{n}\right) .
\end{aligned}
$$

Here $U$ is the sum of the projectile constituent-target optical potentials, assumed central for simplicity. Coordinate $\vec{R}$ is the position of the projectile center-of-mass (c.m.) relative to the target, $T_{R}$ is the corresponding kinetic energy operator 
and $\vec{K}$ is the projectile incident wave number in the c.m. frame, i.e., $E-\varepsilon_{0}=\hbar^{2} K^{2} / 2 \mu$. In writing Eq. (1) $H_{0}$ has been replaced by $\varepsilon_{0}$, it being assumed that the projectile internal energies excited in the reaction are small compared to the total energy $E$, or, that the velocities of the projectile constituents are slow compared to the c.m. translational motion.

The result is a two-body dynamical Schrödinger equation for the $(n+1)$-body problem with only parametric dependence on the projectile internal coordinates. The validity of the adiabatic approximation has been studied extensively in deuteron, ${ }^{6} \mathrm{Li}$, and ${ }^{7} \mathrm{Li}$ induced reactions [4]. Broadly speaking, good agreement is found with more exact three-body methods for projectile energies of order $30 \mathrm{MeV} /$ nucleon. In the following, calculations are presented for ${ }^{11} \mathrm{Be}+{ }^{12} \mathrm{C}$ scattering which solve the three-body adiabatic equation [13] by partial wave expansion and without further approximation, using the method of [14].

In addition to the adiabatic approximation, the few-body Glauber (FBG) models make the semiclassical straight line path assumption. The FBG elastic scattering amplitude for a two-body projectile, with internal wave function $\Phi_{0}(\vec{r})$, is, e.g. [2],

$$
\begin{aligned}
f(\theta)= & -i K \int_{0}^{\infty} b d b J_{0}(q b) \\
& \times\left\{\left\langle\Phi_{0}\left|\exp \left[i \mathcal{X}_{0}^{c}\left(b_{c}\right)+i \mathcal{X}_{0}^{v}\left(b_{v}\right)\right]\right| \Phi_{0}\right\rangle-1\right\},
\end{aligned}
$$

with $q=2 K \sin (\theta / 2)$ the momentum transfer. Here $\mathcal{X}_{0}^{c}$ and $\mathcal{X}_{0}^{v}$ are the eikonal phase shift functions for the core- and valence particle-target systems,

$$
\mathcal{X}_{0}^{i}(b)=-\frac{1}{\hbar v} \int_{-\infty}^{\infty} d z V_{i}\left(\sqrt{b^{2}+z^{2}}\right), \quad(i=c, v),
$$

where $v=\hbar K / \mu$ is the projectile c.m. velocity. The factors $\exp \left(i \mathcal{X}_{0}^{i}\right)$ are related, in the eikonal approximation, to the core- and valence particle-target elastic $S$ matrices $S_{i}\left(b_{i}\right)$, functions of their individual impact parameters. The noneikonal corrections in this two-body projectile case will require modifications to be made to the $\mathcal{X}_{0}^{i}$, or equivalently to the description of the $S_{i}\left(b_{i}\right)$.

Following Wallace [10], and references therein, we make use of the correspondence between the eikonal phase and the expansion of the WKB phase shift. The WKB phase, expressible as an expansion in powers of the parameter $\epsilon=1 / \hbar K v[15]$, reads

$$
\begin{gathered}
\mathcal{X}_{\mathrm{WKB}}^{i}(b)=\sum_{n=0}^{\infty} \frac{\epsilon^{n}}{(n+1) !} \mathcal{X}_{n}^{i}(b), \\
\mathcal{X}_{n}^{i}(b)=-\frac{1}{\hbar v} \int_{-\infty}^{\infty} d z\left(\frac{1}{r} \frac{d}{d r}\right)^{n}\left[r^{2 n} V_{i}^{n+1}(r)\right],
\end{gathered}
$$

for $i=c, v$. We note that the WKB expansion has the eikonal phase shift as its $n=0$ term. The $\mathcal{X}_{\mathrm{WKB}}^{i}$ are not themselves exact. The required correction terms were first studied by Rosen and Yennie [16] and subsequently by Wallace [10].
Our prescription for improving the phases to be used in the FBG models is, therefore, to replace the eikonal phase functions $\mathcal{X}_{0}^{i}$ of Eq. (3) in Eq. (2)

$$
\mathcal{X}_{0}^{i} \rightarrow \mathcal{X}^{i}=\mathcal{X}_{\mathrm{WKB}}^{i}+\mathcal{X}_{\mathrm{RY}}^{i},
$$

where the second term constitutes the Rosen-Yennie (RY) correction terms [16], also expressible as an expansion in powers of $\epsilon$ [10].

As written above, the $\mathcal{X}^{i}$ include fully both Coulomb and nuclear interaction effects since $V(r)=V_{N}(r)+V_{C}(r)$ is the sum of the nuclear and Coulomb interactions. Specifically, the eikonal phase function for a charged core comprises nuclear and Coulomb terms, $\mathcal{X}_{0}^{c}\left(b_{c}\right)=\mathcal{X}_{0 N}^{c}\left(b_{c}\right)+\mathcal{X}_{0 C}^{c}\left(b_{c}\right)$. It is well known that these Coulomb phase terms diverge logarithmically at the limits of the integral over $z$ and we need formally to introduce a screening radius $a_{s}$ which shields the charges at large distances. In the presence of screening [2], we must replace

$$
\mathcal{X}_{0 C}^{c}\left(b_{c}\right) \rightarrow \mathcal{X}_{0 \rho}^{c}\left(b_{c}\right)+\mathcal{X}_{a}^{c},
$$

where $\mathcal{X}_{0 \rho}^{c}$ is the screened Coulomb phase of the core and $\mathcal{X}_{a}^{c}$, proportional to $\eta_{c}$, is a constant. This Coulomb screening need only be applied in the lowest-order term. Elsewhere, $V_{C}$ appears in quadratic or higher powers of $\left(V_{N}+V_{C}\right)$. These terms make only finite range modifications to the integrals over $z$ in higher-order terms. With these clarifications the scattering amplitude reads

$$
\begin{aligned}
f(\theta)= & -i K \int_{0}^{\infty} b d b J_{0}(q b)\left\{\langle \Phi _ { 0 } | \operatorname { e x p } \left[i \overline{\mathcal{X}}^{c}\left(b_{c}\right)\right.\right. \\
& \left.\left.+i \overline{\mathcal{X}}^{v}\left(b_{v}\right)+i \mathcal{X}_{a}\right]\left|\Phi_{0}\right\rangle-1\right\},
\end{aligned}
$$

where $\mathcal{X}_{a}=\mathcal{X}_{a}^{c}+\mathcal{X}_{a}^{v}$ and the $\overline{\mathcal{X}}^{i}$ differ from $\mathcal{X}^{i}$ in that $\mathcal{X}_{0 C}^{i}$ has been replaced by $\mathcal{X}_{0 \rho}^{i}$ in the lowest-order $(n=0)$ term.

To evaluate the integral over $b$ in Eq. (8), it is advantageous, technically, to add and subtract the screened amplitude due to the point Coulomb interaction acting on the projectile c.m. [2], so that

$$
f(\theta)=e^{i \mathcal{X} a}\left\{f_{p t}(\theta)-i K \int_{0}^{\infty} b d b J_{0}(q b) e^{2 i \eta \ln K b}[S(b)-1]\right\} .
$$

The introduction of the screening radius results only in a constant phase factor and the limit $a_{s} \rightarrow \infty$ has no consequences when calculating angular distributions from $f(\theta)$.

All information on the projectile structure and its interactions with the target now appear within the term

$$
S(b)=\left\langle\Phi_{0}\left|\exp \left[i \overline{\mathcal{X}}^{c}\left(b_{c}\right)+i \overline{\mathcal{X}}^{v}\left(b_{v}\right)-2 i \eta \ln K b\right]\right| \Phi_{0}\right\rangle,
$$

the approximate Coulomb modified projectile-target elastic scattering $S$ matrix, which now includes noneikonal modifications within the core-target and valence particle-target two-body systems.

Here we apply the formalism detailed above to the threebody ${ }^{11} \mathrm{Be}+{ }^{12} \mathrm{C}$ system. ${ }^{11} \mathrm{Be}$ is a good example of a binary, ${ }^{10} \mathrm{Be}+n$, single neutron halo nucleus. Our choice of a three- 
body system is to allow comparison of our noneikonal calculations with exact solutions of the three-body adiabatic Schrödinger equation obtained using precisely the same physical inputs. The generalization of the method to fourbody systems is obvious and this application will be presented elsewhere. Fortunately, for ${ }^{11} \mathrm{Be}+{ }^{12} \mathrm{C}$, there are also preliminary elastic scattering data [17] for both the ${ }^{10} \mathrm{Be}$ core and the ${ }^{11} \mathrm{Be}$ composite at 59.4 MeV/nucleon and 49.3 MeV/ nucleon, respectively. While presently these data only extend to c.m. scattering angles of order $10^{\circ}$, they nevertheless provide a valuable constraint on the ${ }^{10} \mathrm{Be}+{ }^{12} \mathrm{C}$ optical potential used and an indication of the importance of noneikonal corrections in relation to expected experimental error bars. Data for a more extended range of scattering angles will be available shortly from GANIL.

Unless otherwise stated, the calculations presented use the following inputs. The core-target, ${ }^{10} \mathrm{Be}+{ }^{12} \mathrm{C}$, optical potential $V_{c} \equiv V_{10}$ was taken as

$$
\begin{aligned}
& V=123.0 \mathrm{MeV}, \quad r_{V}=0.750 \mathrm{fm}, \quad a_{V}=0.80 \mathrm{fm}, \\
& W=65.0 \mathrm{MeV}, \quad r_{W}=0.780 \mathrm{fm}, \quad a_{W}=0.80 \mathrm{fm},
\end{aligned}
$$

with real and imaginary volume Woods-Saxon terms. This potential, consistent with the available data at $59.4 \mathrm{MeV} /$ nucleon, was used at $49.3 \mathrm{MeV} /$ nucleon, the possible weak energy dependence being neglected. The interaction is similar to that used in recent analyses of ${ }^{9} \mathrm{Li}$ and ${ }^{11} \mathrm{Li}$ scattering [18]. The Coulomb interaction was that due to a uniformly charged sphere of radius parameter $r_{c}=1.20 \mathrm{fm}$. The ${ }^{10} \mathrm{Be}$ radius parameters are multiplied by $10^{1 / 3}+12^{1 / 3}$. The valence-target, neutron $+{ }^{12} \mathrm{C}$, optical potential $V_{v} \equiv V_{n}$ is given by the global Becchetti-Greenlees parametrization [19]. The parameters used are tabulated in [2]. The ${ }^{11} \mathrm{Be}$ ground state wave function was taken to be a pure $2 s_{1 / 2}$ neutron single particle state, with separation energy 0.504 $\mathrm{MeV}$, calculated in a central Woods-Saxon potential of geometry $r_{0}=1.00 \mathrm{fm}$ and $a_{0}=0.53 \mathrm{fm}$. Assuming a ${ }^{10} \mathrm{Be}$ core root mean squared (rms) matter radius of $2.28 \mathrm{fm}$, this generates a ${ }^{11} \mathrm{Be}$ composite with rms matter radius of 2.90 $\mathrm{fm}$, in agreement with the most careful recent analysis [20] of halo nucleus sizes. We study the sensitivity of results to this choice of matter radius briefly in the following.

Figure 1 shows the calculated elastic differential crosssection angular distributions (ratio to Rutherford) for ${ }^{11} \mathrm{Be}+{ }^{12} \mathrm{C}$ scattering at $49.3 \mathrm{MeV} /$ nucleon together with the preliminary GANIL data. To assess the importance of breakup and projectile excitation contributions, the dashed curve shows the calculated cross section in the absence of ${ }^{11} \mathrm{Be}$ breakup contributions; that is, the scattering solution of the Schrödinger equation for the single folding model interaction

$$
V_{00}(R)=\left\langle\Phi_{0}\left|V_{10}+V_{n}\right| \Phi_{0}\right\rangle .
$$

The dot-dashed curve shows the results of the conventional lowest-order eikonal model calculation, which includes the effects of intermediate ${ }^{11} \mathrm{Be}$ excitation and breakup channels. The result obtained when using the prescription discussed above, to include the noneikonal corrections to the ${ }^{10} \mathrm{Be}$ and neutron phases, is shown by the long-dashed curve. These, and all calculations shown, include WKB and RY correction

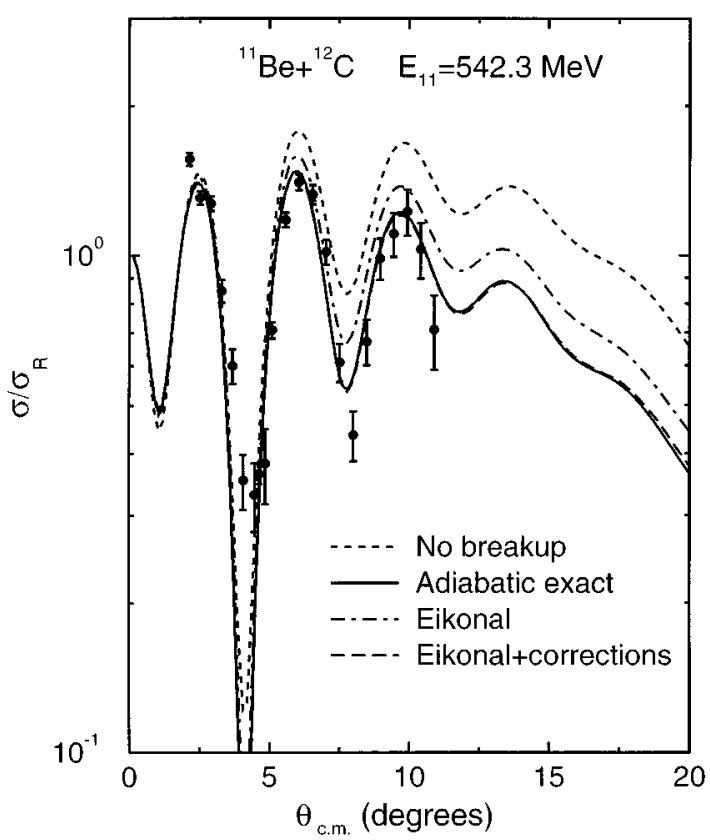

FIG. 1. Calculated ${ }^{11} \mathrm{Be}+{ }^{12} \mathrm{C}$ elastic cross-section angular distributions (ratio to Rutherford) at $49.3 \mathrm{MeV} /$ nucleon together with the available experimental data. The curves show the no breakup (dashed), eikonal (dot-dashed) improved eikonal (long-dashed), and exact adiabatic (solid) calculations.

terms up to and including order $n=3$ in Eqs. (6) and (4). The modified calculations are seen to agree to high precision, out to at least $20^{\circ}$, with the exact adiabatic model calculations, presented by the solid curve. The no breakup, eikonal, noneikonal, and exact calculations are also shown to larger scattering angles in Fig. 2. In addition the lower dot-dashed

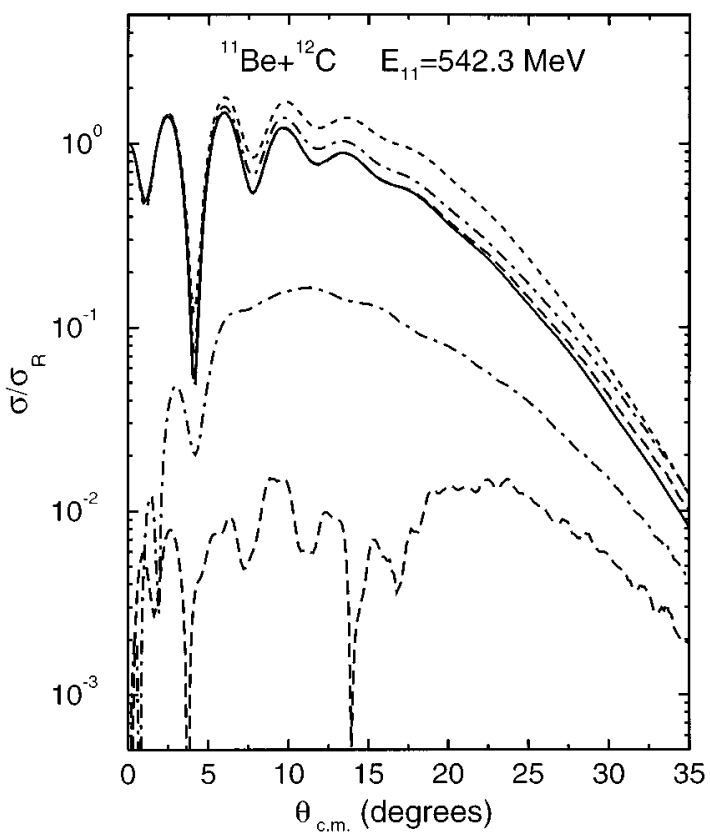

FIG. 2. Calculated ${ }^{11} \mathrm{Be}+{ }^{12} \mathrm{C}$ elastic cross-section angular distributions (ratio to Rutherford) at $49.3 \mathrm{MeV} /$ nucleon. The upper curves are as for Fig. 1. The lower curves show the deviations of the eikonal (dot-dashed) and improved eikonal (long-dashed) calculations from the exact adiabatic calculations. 


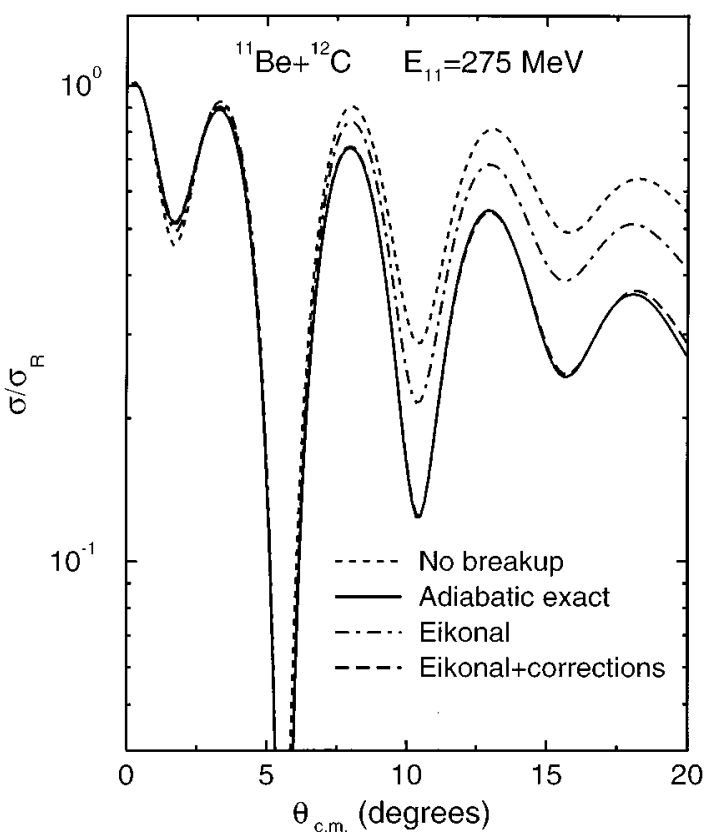

FIG. 3. Calculated ${ }^{11} \mathrm{Be}+{ }^{12} \mathrm{C}$ elastic cross-section angular distributions (ratio to Rutherford) at $25.0 \mathrm{MeV} /$ nucleon. The curves are as for Fig. 1.

and long-dashed curves in Fig. 2 show the computed moduli of the deviations of the lowest-order and improved eikonal model calculations from the full quantum mechanical calculation.

Several points are evident from Figs. 1 and 2. Firstly, projectile excitation and breakup effects are significant and will need to be included in future analyses of experimental data. This observation reinforces the need for accurate and practical methods for the treatment of such processes using few-body models. Secondly, at energies of order $50 \mathrm{MeV} /$ nucleon, there are equally significant discrepancies between the exact and the lowest-order eikonal model calculations, even at the small scattering angles displayed in Fig. 1. Moreover, these deviations are typically greater than the error bars already achieved on available experimental data. Thirdly, the simple prescription proposed here for the inclusion of noneikonal corrections within the few-body Glauber model amplitude, considerably improves the accuracy of the calculated observables. Small departures from the exact calculations are now seen only at c.m. scattering angles beyond $20^{\circ}$. It should be pointed out that these corrections are included at a tiny fraction of the computational expense of carrying out the full partial wave, coupled channels, solution required in the case of the adiabatic Schrödinger equation. Finally, we note that the agreement between the full calculations and the data are very encouraging given the parameter free nature of the three-body calculations.

In Figs. 3 and 4 we present similar calculations, but where we have halved the incident ${ }^{11} \mathrm{Be}$ energy to $25 \mathrm{MeV} /$ nucleon. In lowering the energy, our aim is to enhance the noneikonal contributions. The curves shown have the same meanings as in Figs. 1 and 2; however, there are no experimental data available at this energy. As was expected, the differences between the lowest order eikonal (dot-dashed curve in Fig. 3 ) and the exact adiabatic calculations (solid curves) are significantly larger at this lower energy. However, we note the

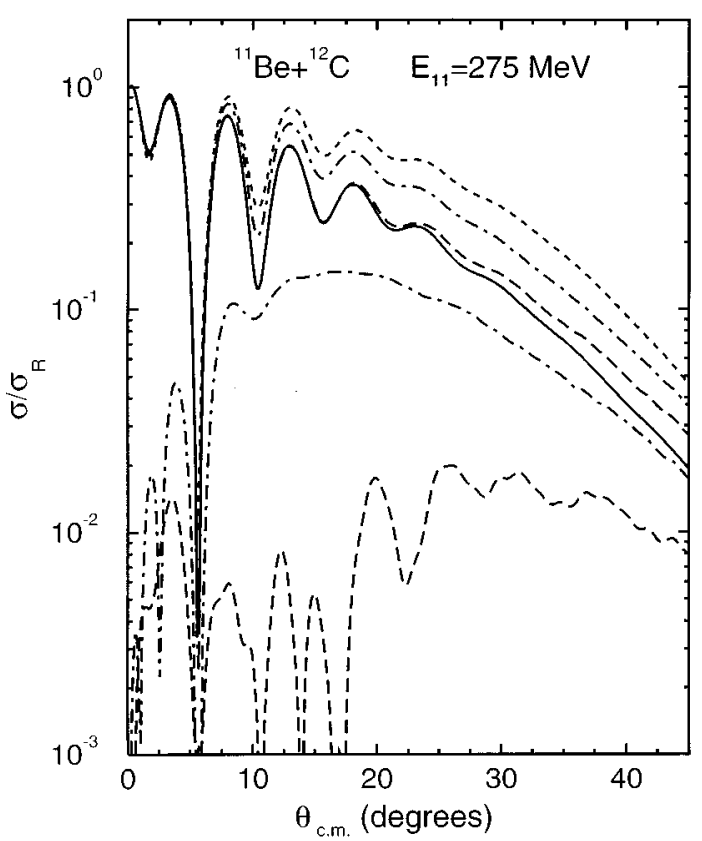

FIG. 4. Calculated ${ }^{11} \mathrm{Be}+{ }^{12} \mathrm{C}$ elastic cross-section angular distributions (ratio to Rutherford) at $25.0 \mathrm{MeV} /$ nucleon. The curves are as for Fig. 2.

continued success of the noneikonal modifications to the few-body amplitude to correct the eikonal calculation very accurately over a significant range of scattering angles. At energies below that presented, the additional adiabatic assumption would itself be suspect and we do not consider applications to lower energies meaningful within the present framework.

It was noted earlier that the structure and interactions of the composite projectile enters the FBG calculation, with or without noneikonal corrections, through the approximation to the composite-target elastic $S$ matrix defined by Eq. (10). This $S$ matrix, apart from simple Coulomb modifications, is generated by taking the average, with respect to the projectile ground state wave function, of the product of all the projectile component-target two-body $S$ matrices. The physical basis of the prescription presented here is, therefore, to use the established WKB and RY corrections to the twobody eikonal phase to systematically improve the description of the two-body $S$ matrices for each constituent channel before the projectile ground state average is carried out. The resulting agreement with the exact adiabatic calculations suggests this simple modification accounts for a large component of the physics included via the exact calculation and provides an economical procedure to extend the range of applicability of the few-body Glauber approach. The noneikonal modifications identified here clearly also have implications for calculated reaction cross sections and calculated breakup momentum distributions at these energies.

A natural expectation, given the explicit spatial averaging in Eq. (10), is that the projectile-target elastic $S$ matrix, and hence the calculated cross section angular distributions, might reflect the spatial extent of the core-valence particle relative motion wave function in a simple way. In Fig. 5 we assess this sensitivity by showing the calculated elastic differential cross-section angular distributions at $49.3 \mathrm{MeV} /$ nucleon for projectile wave functions with rms matter radii 


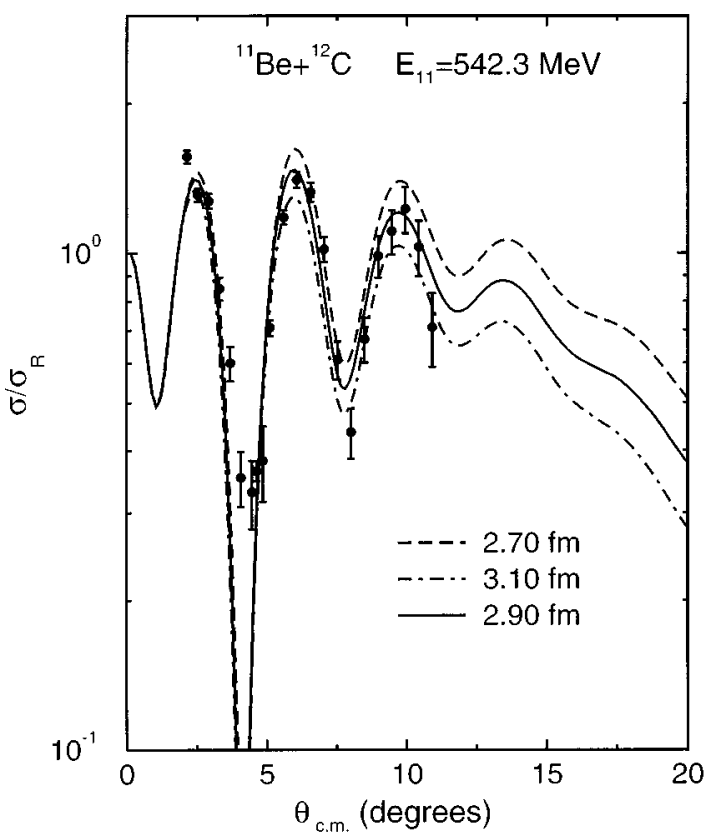

FIG. 5. Calculated ${ }^{11} \mathrm{Be}+{ }^{12} \mathrm{C}$ elastic cross-section angular distributions (ratio to Rutherford) at $49.3 \mathrm{MeV} /$ nucleon for projectile wave functions with rms matter radii of $2.70 \mathrm{fm}$ (long-dashed curve), $2.90 \mathrm{fm}$ (solid curve), and $3.10 \mathrm{fm}$ (dot-dashed curve).

of $2.70 \mathrm{fm}$ (long-dashed curve), $2.90 \mathrm{fm}$ (solid curve), and $3.10 \mathrm{fm}$ (dot-dashed curve). The analysis of reaction crosssection measurements at $800 \mathrm{MeV} /$ nucleon is consistent with the value $2.90 \mathrm{fm}$ [20]. All calculations use the corrected eikonal procedure and also assume the same ${ }^{10} \mathrm{Be}$ core rms matter radius of $2.28 \mathrm{fm}$, so the ground states differ only through the range of the valence neutron-core relative motion wave function. We observe quite strong sensitivity in the angular distribution to the size of the halo particle wave function. Indeed the results shown in Fig. 5 would suggest that elastic scattering data, of comparable quality to those presented, but which extend to larger angles, could provide a very valuable and independent measure of the spatial extent of the halo wave function; that of the single neutron in this case.

To conclude, we have presented a simple physical prescription to extend the range of applicability of Glauber model calculations for few-body systems. This involves including the WKB and RY corrections to the two-body eikonal phase, in each projectile constituent-target two-body channel, prior to the projectile ground state average being carried out. In the two-body projectile case presented, this is shown to result in excellent agreement with exact adiabatic calculations for an expanded and useful range of c.m. scattering angles and for all energies where the adiabatic treatment is applicable. We have shown also that breakup and noneikonal effects are significant even at very forward scattering angles in ${ }^{11} \mathrm{Be}+{ }^{12} \mathrm{C}$ elastic scattering at $49.3 \mathrm{MeV} /$ nucleon. The inclusion of noneikonal corrections leads to a significantly improved description of the available experimental data. We show also that accurate elastic scattering data extending to larger angles may provide an independent measure of the extent of the neutron halo distribution in ${ }^{11} \mathrm{Be}$ and related systems. Full details of the noneikonal corrections, their convergence, the implications of the noneikonal modifications for calculated reaction cross sections at these lower energies, and the application of the method to three-body projectile systems will be presented shortly.

The financial support of the Engineering and Physical Sciences Research Council (U.K.) through grant GR/J95867, and for a Research Studentship Grant (for J.M.B), is gratefully acknowledged. The authors would like to thank Dr. M.D. Cortina-Gil and Dr. P. Roussel-Chomaz, GANIL, Caen, for providing their preliminary experimental data for ${ }^{10,11}$ Be scattering in tabular form, and for their kind permission to use these data in the present analysis.
[1] K. Yabana, Y. Ogawa, and Y. Suzuki, Phys. Rev. C 45, 2909 (1992); Nucl. Phys. A539, 295 (1992).

[2] J.S. Al-Khalili, I.J. Thompson, and J.A. Tostevin, Nucl. Phys. A581, 331 (1995).

[3] Y. Ogawa, Y. Suzuki, and K. Yabana, Nucl. Phys. A571, 784 (1994).

[4] M. Yahiro, Y. Iseri, H. Kameyama, M. Kamimura, and M. Kawai, Prog. Theor. Phys. Suppl. 89, 32 (1986); N. Austern, Y. Iseri, M. Kamimura, M. Kawai, G. Rawitscher, and M. Yahiro, Phys. Rep. 154, 125 (1987).

[5] R.J. Glauber, in Lectures in Theoretical Physics, edited by W.E. Brittin (Interscience, New York, 1959), Vol. 1, p. 315.

[6] L.I. Schiff, Phys. Rev. 103, 443 (1956); D.S. Saxon and L.I. Schiff, Nuovo Cimento 6, 614 (1957).

[7] R.G. Newton, Scattering Theory of Waves and Particles (McGraw-Hill, New York, 1966), p. 575.

[8] R. Blankenbecler and M.L. Goldberger, Phys. Rev. 126, 766 (1962)

[9] R.J. Moore, Phys. Rev. D 2, 313 (1970).

[10] S.J. Wallace, Phys. Rev. D 8, 1846 (1973).
[11] A. Vitturi and F. Zardi, Phys. Rev. C 36, 1404 (1987).

[12] R.C. Johnson and P.J.R. Soper, Phys. Rev. C 1, 976 (1970).

[13] I.J. Thompson, computer program ADIA, Daresbury Laboratory report, 1984 (unpublished).

[14] H. Amakawa, S. Yamaji, A. Mori, and K. Yazaki, Phys. Lett. 82B, 13 (1979).

[15] S.J. Wallace, Ann. Phys. (N.Y.) 78, 190 (1973); Phys. Rev. Lett. 27, 622 (1971).

[16] M. Rosen and D.R. Yennie, J. Math. Phys. (N.Y.) 5, 1505 (1964).

[17] P. Roussel-Chomaz (private communication).

[18] M. Zahar, M. Belbot, J.J. Kolata, K. Lamkin, D.J. Morrissey, B.M. Sherrill, M. Lewitowicz, A.H. Wuosmaa, J.S. Al-Khalili, J.A. Tostevin, and I.J. Thompson, Phys. Rev. C 54, 1262 (1996).

[19] F.D. Becchetti and G.W. Greenlees, Phys. Rev. 182, 1190 (1969).

[20] J.S. Al-Khalili and J.A. Tostevin, Phys. Rev. Lett. 76, 3903 (1996); J.S. Al-Khalili, J.A. Tostevin, and I.J. Thompson, Phys. Rev. C 54, 1843 (1996). 